\title{
Three Dimensional Microstructure Characterization of Polypropylene Blends
}

\author{
Jui-Ching Lin $^{1}$, Ye Huang ${ }^{1}$, Joseph Harris ${ }^{1}$, Brandon Weinlander ${ }^{1}$ and Mary Ann Jones ${ }^{1}$ \\ 1. The Dow Chemical Company, Analytical Sciences, Midland, MI.
}

Polypropylene (PP) is a thermoplastic polyolefin polymer widely used in the automotive industry such as instrument panels and door pillars. PP polymer is often blended with elastomer [1] or fillers [2], respectively, to improve the toughness or increase the stiffness of the material. In order to understand the structure of a multicomponent PP blend or composite system, a three dimensional (3D) microscopy method that has both high spatial resolution and field of view is needed to study both the interfaces of the small discrete phase/phases and also the distribution of the individual phases.

In this study, a combination of focused ion beam - scanning electron microscopy (FIB-SEM) with heavy metal staining is applied to study the 3D structures of a PP blend (70\% poly(propylene) homopolymer and $30 \%$ polyolefin elastomer) and a PP blend filled with talc (poly(propylene) homopolymer, 30\% polyolefin elastomer, and $20 \%$ talc). For the PP blend filled with talc (TF-PP), the sample was notched then fractured at $-25^{\circ} \mathrm{C}$ at a rate of $3.4 \mathrm{~m} / \mathrm{s}$. Both the samples were vapor stained with $\mathrm{RuO}_{4}$ [3]. The samples were serially sectioned and imaged using FIB-SEM instrument. A layer of material $20 \mathrm{~nm}$ (PP blend) or $25 \mathrm{~nm}$ (TF-PP) thick was removed each time during the auto slice and view process. The collected image stacks were aligned and analyzed using Fiji [4] and FEI Avizo software. Although originally both the samples were non-conductive and beam sensitive under the $\mathrm{Ga}^{+}$ion beams, the samples became conductive enough that no charging problems were observed during automatic slice and view process after the $\mathrm{RuO}_{4}$ treatment. The samples became hard and beam insensitive that no beam damaged was observed after continuously milling and imaging the samples for 4 days using $30 \mathrm{KeV}, 47$ $\mathrm{nA} \mathrm{Ga}^{+}$ions and $2 \mathrm{KeV}, 1.6 \mathrm{nA}$ electrons. The high loading of hard talc in TF-PP sample did not cause a problem to ion beam cutting and polishing.

The measurement of the elastomer particle size and shape [5] of the PP blend sample shows that it has many small particles, about 50 volume $\%$ of the particles are smaller than $1 \mu \mathrm{m}^{3}$. Particle shape measurement using "sphericity" shows that not all the particles are spheres. Because the shape of small and smooth objects are difficult to approximate with discrete cube-shaped voxels, it was found that smaller particles in the blend sample, size smaller than $0.05 \mu \mathrm{m}^{3}$, can unrealistically have sphericity greater than one when spatial resolution is $25 \mathrm{~nm}$. The dispersion of the elastomer particles were studied from two sub volumes of the PP blend (Figure 1) and the results show that the dispersion is not uniform across the whole sampling volume. The results demonstrated that by combining image analysis with the FIB-SEM microscopy method, structural information of a PP blend material, such as the size, shape, and dispersion of the different phases, can be quantitatively characterized in 3D.

For the TF-PP sample, the cross-section images were perpendicular to the direction of crack growth and images were collected along the direction of the crack (Figure 2). $\mathrm{RuO}_{4}$ preferentially stains the elastomer phase, so that phase appears brighter than the inorganic talc fillers due to the Ru metal. Some portion of the crack is bright due to the residue of $\mathrm{Ru}$ stain. The images clearly show three dimensional deformation and orientation of the soft elastomer particles near the crack. The microscopy method allows us to view the structure of fractured PP materials in great detail and in 3D near the crack and to estimate the volume of material involved in deformation. It also helps us understand the sequencing of 
events giving insight into deformation and failure mechanisms [6].

References:

[1] S. M. Zebarjad et al., Journal of Applied Polymer Science, 90(14), (2003), p. 3767.

[2] D. Arencon, and J. I. Velasco, Materials, 2(4), (2009), p. 2046.

[3] J. S. Trent, J. I. Scheinbeim, and P. R. Couchman, Macromolecules, 16(4), (1983), p. 589.

[4] J. Schindelin, et al., Nat Meth, 9(7), (2012), p. 676.

[5] J. Ollion, et al., Bioinformatics, 29(14), (2013), p. 1840.

[6] The authors would like to acknowledge Robert Cieslinski and Jeff Munro for valuable discussions. The authors also like to thank Michio Ono for the blend sample.

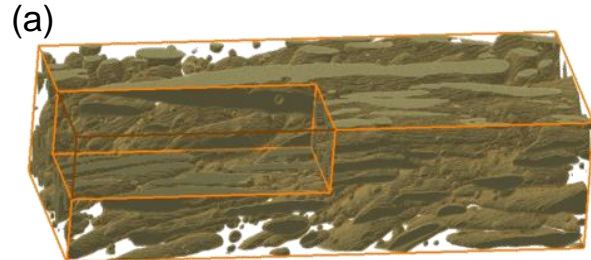

(c)

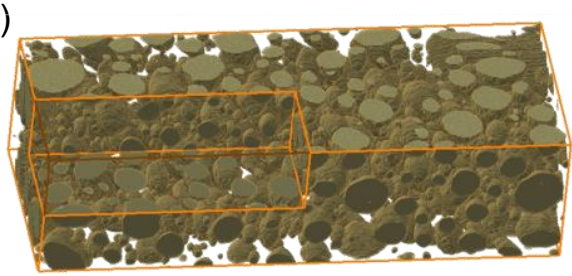

(b)

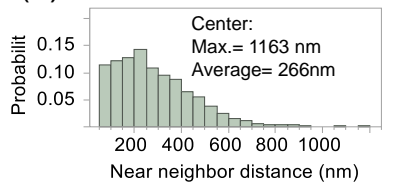

(d)

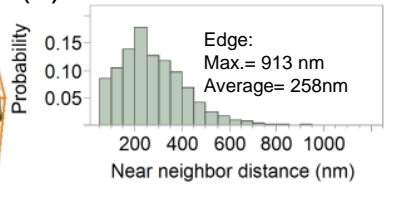

Figure 1. (a) \&(c) 3D rendering of the elastomer phase in the (a) center and (c) edge regions of the PP blend sample with a corner removed for the purpose of clarity. Width of the regions is $18.8 \mu \mathrm{m}$. (b)\&(d) Near neighbor distances of the elastomers in the center region and the edge region, respectively.

(a)

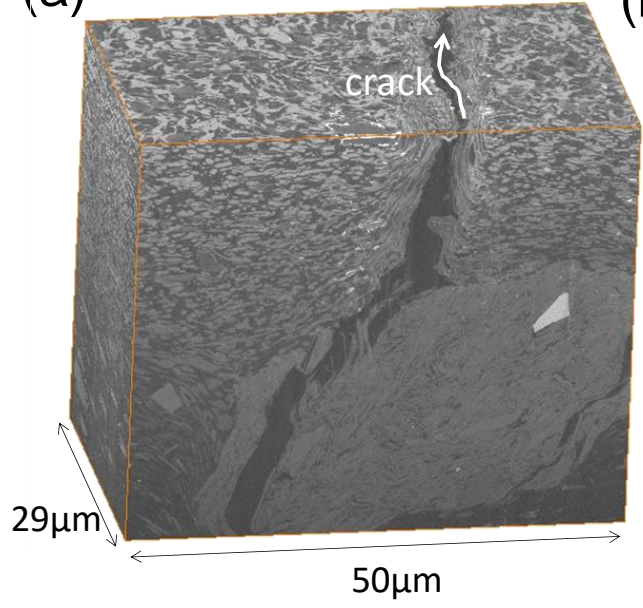

(b)

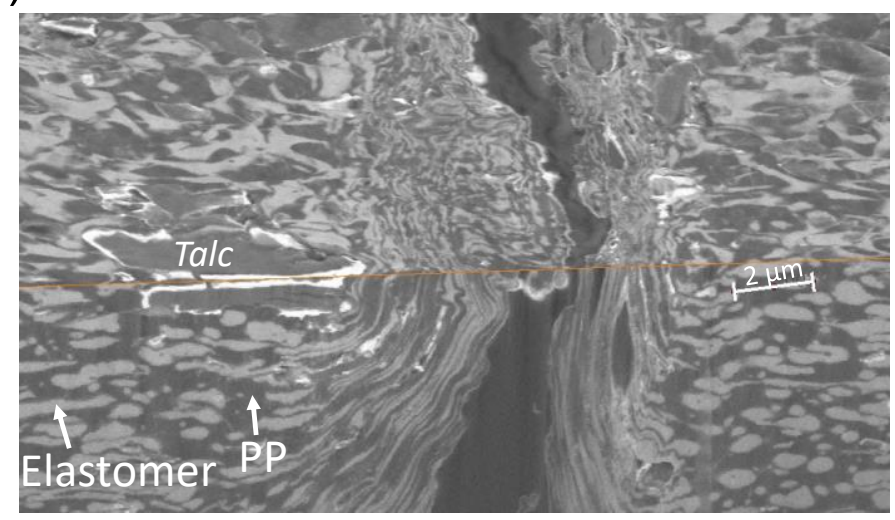

Figure 2. 3D structure of the TF-PP sample. (a) The structure is visualized using orthogonal planes (b) High magnification section of (a). 